\title{
An Eigenvector Based Method for Point Source Localization in Blurred Images
}

\author{
Metin Gunsay and Brian D. Jeffs, members IEEE
}

\begin{abstract}
Brigham Young University, Department of Electrical and Computer Engineering
459 Clyde Building. Provo Utah 84602. (801) 378-3062. email: metin, bjeffs@ee.byu.edu.
\end{abstract}

\begin{abstract}
In this paper we address the problem of resolving and localizing blurred point sources in intensity images. A new approach to image restoration is introduced which is a 2-D generalization of techniques originating from the field of direction of arrival estimation (DOA). It is shown that in the frequency domain, blurred point source images can be modeled with a structure analogous to the response of linear sensor arrays to coherent sources. Thus the problem may be cast into the form of DOA estimation, and modern eigenvector based subspace decomposition algorithms, such as MUSIC, may be adapted to search for these point sources. A generalization of array smoothing based on a regularization operator is introduced for 2-D arrays in order to achieve rank enhancement in the signal space of the covariance matrix.
\end{abstract}

\section{Introduction}

Point source localization (PSL as used in this work) is the problem of resolving individual points. or impulses, in a discrete data sequence, or image that have been corrupted by convolution with some finite support blurring function. It has been addressed in various forms [1]-[5]. PSL is pertinent to the fields of astronomical image restoration, biomedical imaging and echo resolution. Deblurring of star fields is one of the major applications of PSL in images. Blur in long exposure astronomical star images may be due to atmospheric turbulence, misfocus, poor telescope tracking. finite aperture size. or other optical distortion effects. Atmospheric turbulence can cause nearby stars to become blurred beyond resolution so as to appear as a single star. We will address the 2-D case, but the algorithm may be extended to 1-D and higher dimensional data.

The PSL problem for a blurred image $M_{1} \times M_{2}$ a "snap shot" at time $t$ can be formulated in the following linear model:

$g(t)=D f+n(t)$ where $g$ is the colexigraphically ordered (rowscanned) observation vector of length $M_{1} \times M_{2}=M, D$ is an $M \times M$ circulant convolution matrix whose columns are formed from spatially shifted copies of the point spread function (PSF), $f$ is the desired uncorrupted deterministic image vector of length $M$, and $n$ is the additive observation noise vector. It is assumed that the noise is uncorrelated among the elements. We may make the assumption that the columns of $D$ are circularly shifted copies of one another if we assume that points that we are trying to locate are away from boundaries of our image.

The new algorithm casts the PSL problem into a new form which can be viewed as a coherent source direction of arrival estimation problem. The benefits of any advances in the field of DOA estimation can be applied directly to this problem. Because our algorithm is based on eigenvector techniques it naturally inherits the property of super-resolution or interpixel resolution of points. We do not assume that the source amplitudes are time varying in order to build up rank in the signal space of our covariance matrix as other authors have assumed [4][5]. but introduce a new method of 2-D rank enhancement which is a generalization of the array smoothing technique [8]. Our method has an added benefit of allowing regularization of the spatial smoothing.

\section{Subspace Methods and Spatial Smoothing}

The usual observation model applied to DOA estimation is

$\boldsymbol{x}=\boldsymbol{A u}+\boldsymbol{\eta}$.

where $\boldsymbol{x}$ is the observed array data, $A$ has columns corresponding to the array response for each of the $P$ sources. and $\eta$ is additive noise. Subspace DOA methods exploit the special structure of the covariance matrix $\boldsymbol{R}$ of the observed array data, by decomposing it into orthogonal subspaces. Eigenvector algorithms decomposes $\boldsymbol{R}$ into two orthogonal subspaces: $\boldsymbol{E}_{s}$ and 
$\boldsymbol{E}_{n}$, the "signal" and "noise subspaces" respectively, by solving the generalized eigenvector problem

$\boldsymbol{R}\left[\boldsymbol{E}_{s} \mid \boldsymbol{E}_{n}\right]=\boldsymbol{\Lambda} \Sigma\left[\boldsymbol{E}_{s} \mid \boldsymbol{E}_{n}\right]$,

where $A$ is the diagonal eigenvalue matrix with the eigenvalues in descending order and $\Sigma$ is the noise convariance matrix. Since $\boldsymbol{E}_{s}$ and $\boldsymbol{A}$ span the same subspace and $\boldsymbol{E}_{n} \perp \boldsymbol{E}_{s}$, it is also true that $\boldsymbol{E}_{n} \perp \boldsymbol{A}$. The algorithm searches for the steering vectors, $\boldsymbol{a}(\theta)$, which are most orthogonal to the noise space, and these vectors correspond to the directions of arrival of the sources.

The MUSIC algorithm is a common method that exploits this orthogonality property [6]. The MUSIC spectrum is defined as

$$
\mathrm{P}_{M U}(\theta)=\frac{a(\theta)^{H} a(\theta)}{a(\theta)^{H} E_{n} E_{n}{ }^{H} a(\theta)},
$$

where $a(\theta)$ is a proposed steering vector at angle $\theta$. The $\theta$ values which correspond to the peaks of the spectrum, $\mathrm{P}_{M U}(\theta)$, are the true direction of arrival angles.

Simple eigenvector based methods fail if the sources are coherent, because the rank of $R$ is less than $P$. However, this may be overcome in uniformly spaced line arrays using spatial smoothing [8]. This method increases the rank of the signal space of $\boldsymbol{R}$ by averaging together the autocovariance matrices of shorter subarrays, and takes advantage of the fact that the data in each subarray is just a phase shifted copy of the other subarrays. Pillai offers an excellent tutorial on eigenvector based methods and spatial smoothing [7].

\section{Theoretical Development}

In this section we transform the problem expressed by (1) into a problem which can be solved with traditional DOA estimation techniques.

\subsection{Frequency Domain Signal Model}

Since $f$ is sparse we may replace $D f$ with $A \boldsymbol{u}$, where $\boldsymbol{u}$ contains all of the intensity coefficients of all the nonzero elements of $f$, and $A$ contains only those columns of $D$ which correspond to the elements of $u$ :

$$
\boldsymbol{g}(t)=\boldsymbol{A u}+\boldsymbol{n}(t) \text {. }
$$

This differs from the DOA problem in two ways: 1) $u$ which contains the magnitudes of the sources is not time varying, and 2) the columns of $\boldsymbol{A}$ are not cisoids but are shifted versions of the blur function which is of finite support. In order to map spatial shifts in source position to phase shifts, (5) can be transformed into the frequency domain by multiplying $g(t)$ by $\mathcal{F}$, a truncated version of the 2-D DFT matrix. The observed image is real and its 2-D DFT has conjugate symmetry, thus half of the elements contain redundant information, and the order of the system can be reduced by half without loss of resolution. The truncated 2-D DFT matrix is formed from the upper $N=M / 2$ rows of the standard [9] unitary DFT matrix $\mathcal{F}^{\prime}$ which is defined as

$\mathcal{F}^{\prime}=F^{\text {row }} \otimes F^{\text {col }}$,

where $\otimes$ is a Kronecker product, and $F^{\text {row }}$ and $\boldsymbol{F}^{\text {col }}$ are the 1-D DFT matrices whose elements are $F_{i, k}^{\text {row }}=e^{-j 2 \pi m n / M_{1}}, \quad M_{1} / 2 \leq i, k \leq M_{1} / 2-1 \quad$ and $F_{i, k}^{c o l}=e^{-j 2 \pi k / M_{2}}, \quad M_{2} / 2 \leq i, k \leq M_{2} / 2-1$, respectfully. Thus $\mathcal{F}$ is $M \times N$ and is defined as:

$\mathcal{F}_{i, k}=\mathcal{F}_{i, k}^{\prime}$

for $0 \leq i \leq N$ and $0 \leq k \leq M$. Multiplying a vector containing a row scanned image by $\mathcal{F}$ results in a frequency unwrapped image, a condition which is necessary for fractional pixel resolution. The frequency domain version of (5) now becomes

$\mathcal{F g}(t)=\mathcal{F} \boldsymbol{A} \boldsymbol{u}+\mathcal{F} \boldsymbol{\eta}(t)$

$\widetilde{g}(t)=\widetilde{A} \boldsymbol{u}+\tilde{\eta}(t)$

where $\sim$ designates the 2-D DFT of the row scanned images contained in $g(t), n(t)$ and each of the columns of the matrix $A$. The vector $\tilde{g}(t)$ has complex elements and length $N$. Since $\boldsymbol{u}$ is independent of the time index, equation (7) is in the form of a coherent DOA problem. All the columns of $\tilde{\boldsymbol{A}}$ have the same magnitude, but are phase shifted versions of one another, because the columns of $\boldsymbol{A}$ are spatially shifted versions of the blurring function. We can express $\tilde{\boldsymbol{A}}$ as the product of a diagonal matrix $\boldsymbol{H}$ whose elements correspond to the frequency domain PSF, and a matrix $V$ which contains the phase information for each of the columns of $\tilde{\boldsymbol{A}}$ :

$\tilde{A}=\boldsymbol{H}\left[v_{x_{1}, y_{1}}\left|v_{x_{2}, y_{2}}\right| \cdots \mid v_{x_{p}, y_{p}}\right]=H V$.

The column vectors $v_{x_{p}, y_{p}}$ of $V$ correspond to images with a single unblurred point at location $\left(x_{p}, y_{p}\right)$ which have been transformed into the frequency domain, and are expressed as

$$
\begin{aligned}
\boldsymbol{v}_{x, y}= & {\left[e^{-j 2 \pi\left(\frac{0 x}{M_{1}}+\frac{0 y}{M_{2}}\right)}, e^{-j 2 \pi\left(\frac{0 x}{M_{1}}+\frac{1 y}{M_{1}}\right)}, \cdots, e^{-j 2 \pi\left(\frac{O x}{M_{1}}+\frac{\left(M_{2}-1\right) y}{M_{2}}\right)},\right.} \\
& e^{-j 2 \pi\left(\frac{1 x}{M_{1}}+\frac{0 y}{M_{2}}\right)}, e^{-j 2 \pi\left(\frac{1 x}{\left.M_{1}+\frac{1 y}{M_{2}}\right)}, \cdots, e^{-j 2 x\left(\frac{1 x}{M_{1}}+\frac{\left(M_{2}-1\right) y}{M_{2}}\right)}, \cdots\right.} \\
& \left.e^{-j 2 \pi\left(\frac{\left(M_{1}-1\right) x}{M_{1}}+\frac{0 y}{M_{2}}\right)}, \cdots, e^{-j 2 \pi\left(\frac{\left(M_{1}-1\right) x}{M_{1}}+\frac{\left(M_{2}-1\right) y}{M_{2}}\right)}\right]^{T}
\end{aligned}
$$




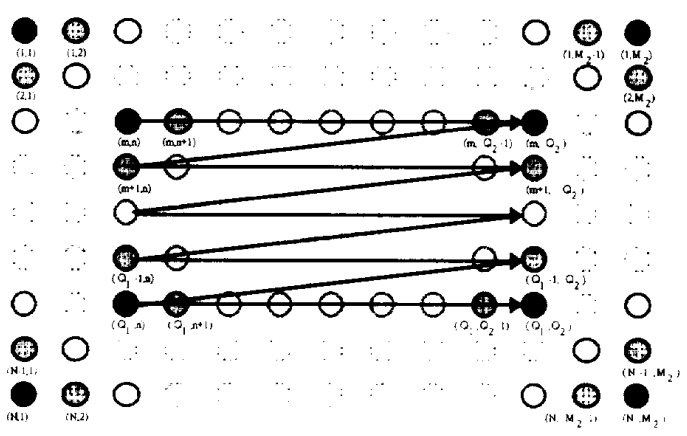

Figure 1. Row scanned subimage at $(m, n)$.

Substituting (8) into (7),

$\widetilde{\boldsymbol{g}}(t)=\boldsymbol{H} \boldsymbol{u}+\tilde{\boldsymbol{\eta}}(t)$

yielding an autocovariance matrix of

$\boldsymbol{R}_{\widetilde{g}}=\boldsymbol{H} \boldsymbol{V} \boldsymbol{R}_{u} \boldsymbol{V}^{H} \boldsymbol{H}^{H}+\boldsymbol{I} \sigma_{\eta}^{2}$,

which is clearly of the same form as the DOA problem. The above formulation assumes that each of the blurred point sources in the observed image have integer pixel shifts in relation to the PSF. If the points in the blurred image have shifts which are a fraction of a pixel in relation to the PSF then (10) is a close approximation. The affects of fractional pixel shifts on this approximation will be discussed in the following section.

\subsection{Generalized 2-D Array Smoothing}

The signal space of $\boldsymbol{R}_{\bar{g}}$ is of rank one because the magnitudes of the point sources are not time varying, resulting in a coherent problem. In addition, the elements in the data array are non-uniformly weighted by $\boldsymbol{H}$. The rank of the signal space must be increased to $P$ for eigenvector based methods to work and this can be accomplished by a new technique which is a generalization of spatial smoothing [8]. Rank enhancement is accomplished by averaging in the frequency domain over the autocovariance matrices of subimages. If we average vertically across an image by $L_{1}$ shifts and horizontally by $L_{2}$ shifts, then a total of $L=L_{1} L_{2}$ subimages may be averaged together to build the sample covariance matrix.

Figure 1 shows how the subimages are extracted from a larger image and how they are arranged into a vector. We will now introduce some notation that will aid us in annotating the colexigraphically ordered subimages. Note that in (10) each of the columns of $V$, the diagonal of $\boldsymbol{H}$, and the column vectors $\boldsymbol{g}$ and $\boldsymbol{n}$ are colexigraphically ordered images. The subscript $[m, n]$ will be applied to these variables to refer to the subimage whose upper left corner is at the position $(m, n)$ in the corresponding 2-D images. The subimages are of size $\Theta$ ${ }_{1} \mathrm{x} \Theta_{2}$ where $\Theta_{1}=N-L_{1}+1$ and $\Theta_{2}=M_{2}-L_{2}+1$.

For example, in the case of the image contained in $\tilde{\mathrm{g}}(t)$ of $(10)$ the subimage vectors are then defined as:

$$
\begin{aligned}
\widetilde{\boldsymbol{g}}_{[m, n]}(t)= & {\left[\widetilde{\boldsymbol{g}}_{m, n}, \widetilde{\boldsymbol{g}}_{m, n+1}, \cdots, \widetilde{\boldsymbol{g}}_{m, \Theta_{2}},\right.} \\
& \widetilde{\boldsymbol{g}}_{m+1, n}, \widetilde{\boldsymbol{g}}_{m+1, n+1}, \cdots, \widetilde{\boldsymbol{g}}_{m+1, \Theta_{2}}, \cdots, \\
& \left.\widetilde{\boldsymbol{g}}_{\Theta_{1}, n}, \widetilde{\boldsymbol{g}}_{\Theta_{1}, n+1}, \cdots, \widetilde{\boldsymbol{g}}_{\Theta_{1} \Theta_{2}}\right]^{T}
\end{aligned}
$$

Using this notation we can express any subimage as

$\widetilde{\boldsymbol{g}}_{[m, n]}(t)=\boldsymbol{H}_{[m, n]} V_{[m, n]}+\widetilde{\boldsymbol{\eta}}_{[m, n]}(t)$,

$V$ is not Vandermonde as it is in the above formulation of array smoothing. but fortunately there exists a simple relationship between the all of the $V_{[m, n]}$. We can express the $V_{[m, n]}$ in terms of the product of $V_{[1,1]}$ and the diagonal matrices $C^{m, n}$ whose elements are $\operatorname{diag}\left(C^{m, n}\right)=\left[e^{-j 2 \pi\left(\frac{x_{1}(m-1)}{M_{1}}+\frac{y_{1}(n-1)}{M_{2}}\right)}\right.$,
$\left.e^{-j 2 \pi\left(\frac{x_{2}(m-1)}{M_{1}}+\frac{y_{2}(n-1)}{M_{2}}\right)} \cdots, e^{-j 2 \pi\left(\frac{x_{p}(m-1)}{M_{1}}+\frac{y_{P}(n-1)}{M_{2}}\right)}\right]$.

Thus

$V_{[m, n]}=V_{[1,1]} C^{m, n}$

Equation (12) is now expressed as

$\widetilde{\boldsymbol{g}}_{\left[m, n_{-}\right.}(t)=\boldsymbol{H}_{[m, n]} V_{[1,1]} C^{m, n} \boldsymbol{u}+\widetilde{\eta}_{[m, n]}(t)$,

and assuming the noise is uncorrelated from pixel to pixel its autocovariance matrix is

$$
\begin{aligned}
\boldsymbol{R}_{[m, n]} & =\boldsymbol{H}_{[m, n]} \boldsymbol{V}_{[1,1]} C^{m . n} \boldsymbol{R}_{u}\left(C^{m, n}\right)^{H} \boldsymbol{V}_{[1,1]}^{H} \boldsymbol{H}_{[m, n]}^{H}+ \\
& I \sigma_{\eta}^{2}
\end{aligned}
$$

Before these subimages can be averaged, the modulation introduced by $\boldsymbol{H}$ must be removed. An arbitrary diagonal regularization matrix, $Q$, may be chosen and corresponding weighting matrices, $S_{[m . n]}$, are computed such that $\boldsymbol{Q}=\boldsymbol{S}_{[m, n]} \boldsymbol{H}_{[m, n]} \forall[m . n]$, then

$$
\begin{aligned}
\boldsymbol{R}= & \frac{1}{L} \sum_{\forall m, n} \boldsymbol{S}_{[m, n]} \boldsymbol{R}_{[m, n]} \boldsymbol{S}_{[m, n]}^{\mathrm{H}} \\
= & \boldsymbol{Q} \boldsymbol{V}_{[1,1]}\left[\frac{1}{L} \sum_{\forall m, n} C^{m, n} \boldsymbol{R}_{\boldsymbol{u}}\left(C^{m, n}\right)^{H}\right] \boldsymbol{V}_{[1,1]}^{\mathrm{H}} \boldsymbol{Q}^{\mathrm{H}}+ \\
& \frac{\sigma_{\eta}^{2}}{L} \sum_{\forall m, n}\left|\boldsymbol{S}_{[m, n]}\right|^{2}
\end{aligned}
$$




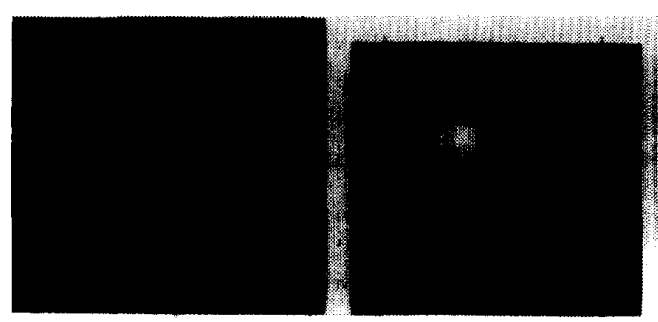

(a)

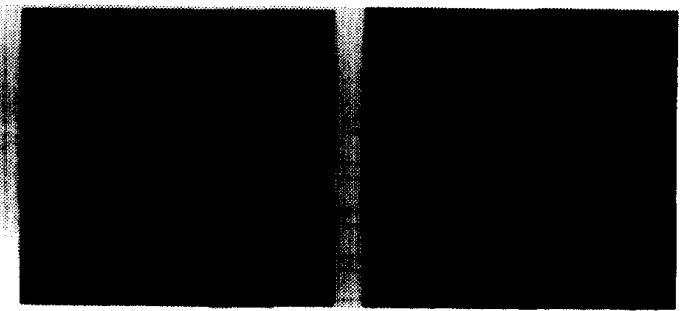

(c)

(d)

Figure 2. Synthetic star example. a) unblurred Image. b) blurred image with 40dB SNR. c) MUSIC spectrum. d) peaks of spectrum.

$$
=Q V_{[1,1]} \bar{R}_{u} V_{[1,1]}^{\mathrm{H}} Q^{\mathrm{H}}+\sigma_{\eta}^{2} \overline{\boldsymbol{S}}
$$

The $\boldsymbol{S}_{[m, n]}$ allow the $\boldsymbol{H}_{[m, n]}$ to be pulled out of the summation. As in the traditional DOA problem it can be shown that the rank $\overline{\boldsymbol{R}}_{u}$ will increase to $P$ for every additional subarray which is averaged in (see Appendix A), if subimages from the diagonal are used in the averaging. We shall refer to $Q$ as the smoothing regularization matrix.

\subsection{Restoration Algorithm}

The matrix $Q$ is selected depending upon the type of regularization desired, and the appropriate weighting matrices $S_{[m, n]}$ are computed. The sample covariance matrix is computed as an estimate of $\boldsymbol{R}$

$\hat{\boldsymbol{R}}=\frac{1}{L \Omega} \sum_{\forall[m, n]} \boldsymbol{S}_{[m, n]}\left(\sum_{l=1}^{\Omega} \widetilde{\boldsymbol{g}}_{[m, n]}(I T) \widetilde{\boldsymbol{g}}_{[m, n]}^{H}(I T)\right) \boldsymbol{S}_{[m, n]}^{H}$

where $T$ is the sample interval between snapshots. The algorithm is suitable for the single or multiple snapshot cases. If multiple snapshots are available, then a more accurate estimate of $\boldsymbol{R}$ is obtained. If only one is available and the image is of sufficient size so that the number of subimages is greater than the number of sources, then (19) will provide a estimate of $R$ with necessary rank to render a signal subspace of full rank.

Estimates of the signal and noise subspaces, $\hat{\boldsymbol{E}}_{s}$ and $\hat{E}_{n}$, are found by solving the generalized eigenvector problem:

$\hat{\boldsymbol{R}}\left[\hat{\boldsymbol{E}}_{s} \mid \hat{\boldsymbol{E}}_{n}\right]=\hat{\boldsymbol{\Lambda}} \overline{\boldsymbol{S}}\left[\hat{\boldsymbol{E}}_{s} \mid \hat{\boldsymbol{E}}_{n}\right]$

$$
P(x, y)=\frac{\left(Q v_{x, y}\right)^{H}\left(Q v_{x, y}\right)}{\left(Q v_{x, y}\right)^{H} \hat{E}_{n} \hat{\boldsymbol{E}}_{n}{ }^{H}\left(Q v_{x, y}\right)}
$$

where $v_{x, y}$ is the position vector corresponding to a point at location $(x, y)$. Once source locations are found, a simple least squares fit can be made for the amplitudes of the peaks, because solving for the location and amplitude are separable problems [5].

\section{Results}

Figure 1 demonstrates the case of deblurring a synthetic star cluster using eigenvector based methods. The star image is blurred by a Gaussian shaped PSF and i.i.d. Gaussian noise is added for a SNR of $40 \mathrm{~dB}$, where

$S N R=10 \log \frac{\sigma_{g}^{2}}{\sigma_{\eta}^{2}}$

and $\sigma_{g}^{2}$ is the variance of the observed image. The upper-left cluster contains two stars which are separated by only one half of a pixel. Figure 1.c is the processed image showing the MUSIC spectrum at a resolution of 4 times the original image. Figure 1.d shows the locations of the peaks of the MUSIC spectrum. Note that the original source positions are located correctly and that high resolution is obtained. The $Q$ used in the example is

$Q_{i, i}=\min _{m, n}\left(\left(\boldsymbol{H}_{[m, n]}\right)_{i, i}\right)$

In addition, the values of $Q$ corresponding to certain high frequency regions of the $\mathrm{H}$ which have large modeling error were set to zero. 


\section{Conclusions}

We have shown that there exists a duality between the PSL and the coherent DOA problems. Indeed, if the point sources are not blurred the two problems are frequency domain duals of one another. In the coherent DOA problem the aim is to determine the linear combination of cisoids that are superimposed on the linear array, while in PSL it is to determine the linear combination of shifts of a particular finite support function. Transforming the PSL problem into the frequency domain causes the spatial shifted blurred points to become a linear combination of cisoids modulated by the frequency domain blurring function. The benefit of showing the duality of the problems is that advances in one field can be applied to the other. Eigenvector based techniques are desirable because they allow for super-resolution. approximate the ML estimate, and are computationally tractable.

Since the resulting frequency domain problem is coherent, a means of rank enhancement of the covariance function was introduced. This technique is a generalization of smoothing because it introduces a regularization operator $Q$ with its associated weighting matrices $\boldsymbol{S}$. These weighting matrices compensate for the affects of the modulation introduced by the blurring function. and allow regularization to reduce noise amplification in the system which is caused by the division of small values of the frequency domain blurring function. Model error from fractional pixel shifts in the blurring function can also be reduced by proper selection of $Q$. Previously, rank enhancement through smoothing had only been treated for 1-D uniform linear arrays. Our technique generalizes this to the case of arbitrarily placed elements on a uniform 2-D lattice.

Our algorithm could also be particularly useful in the $1-D$ case of finding the location of overlapping echoes. There is no requirement that the sources be time varying, and the case of data consisting of a single snap-shot is easily dealt with.

\section{Appendix A}

In this appendix we wish to prove that generalized smoothing in (15) leads to a covariance matrix $\overline{\boldsymbol{R}}_{u}$ of rank $P$, if $L \geq P$ and if the averaging is done along the diagonal of the observed image, i.e. $m=n$ in $\overline{\boldsymbol{R}}_{[m, n]}$. For the fully coherent case $\boldsymbol{R}_{u}=\alpha \alpha^{H}$. thus $\overline{\boldsymbol{R}}_{u}=\boldsymbol{B} \boldsymbol{B}^{H}$. where

$\boldsymbol{B}=\left[\boldsymbol{\alpha}\left|\alpha \boldsymbol{C}^{1.1}\right| \cdots \mid \boldsymbol{C}^{L, L} \boldsymbol{\alpha}\right]$.
The matrix $B$ may then be separated into the product of a diagonal matrix and a Vandermonde matrix

$$
\boldsymbol{B}=\left[\begin{array}{cccc}
\alpha_{1} & & & \boldsymbol{0} \\
& \alpha_{2} & & \\
& & \ddots & \\
0 & & & \alpha_{P}
\end{array}\right]\left[\begin{array}{ccccc}
1 & w_{1}^{1} & w_{1}^{2} & \cdots & w_{1}^{L-1} \\
1 & w_{2}^{1} & w_{2}^{2} & \cdots & w_{2}^{L-1} \\
\vdots & \vdots & \vdots & \ddots & \vdots \\
1 & w_{P}^{1} & w_{P}^{2} & \cdots & w_{P}^{L-1}
\end{array}\right]
$$

where

$w_{p}=e^{-j 2 \pi\left(\frac{x_{p}}{M} \cdot \frac{y_{p}}{M}\right)}$.

Clearly the rank of $\boldsymbol{B}$ is the minimum of $P$ and $L$. If the number of vertical subimages is less than $P$ and if averaging is done across other sections of the image in addition to the diagonal, then the rank may or may not increase depending upon the location of the sources. For example it is easy to show that if the stars are all located in a single row then smoothing across a set of vertical subarrays will not increase the rank of $\overline{\boldsymbol{R}}_{u}$. While averaging in off-diagonal subimages may not increase rank, it is useful in creating a better estimate of $\boldsymbol{R}$.

\section{References}

[1] B.J. Jeffs, M. Gunsay and J. Dougal, "Resolution Enhancement of Blurred Star Field Images by Maximally Sparse Restoration", SPIE, San Diego, Vol. 1567, pp 511-21, 1991.

[2] B.J. Jeffs and M. Gunsay, "Restoration of Blurred Star Field Images by Maximally Sparse Optimization" IEEE Transactions on Image Processing, Vol. 2, No. 2, pp. 202-11, Apr. 1993

[3] K.A. Marsh and J.M. Richardson, "The objective function implicit in the clean algorithm". Astron. Astrophys., Vol. 182, pp. 17-178, 1987.

[4] A. M. Bruckstein, T. Shan and T. Kailath, "The Resolution of Overlapping Echoes", IEEE Transactions on Acoustics, Speech and Signal Processing, Vol. 33, No. 6, pp. 1357-67, Dec. 1985.

[5] J. C. Mosher P, P. S. Lewis, and R. Leahy, "Multiple Dipole Modeling and Localization from Spatio-Temporal MEG Data", IEEE Transactions on Biomedical Engineering, Vol. 39, pp. 541-57 June 1992.

[6] R. 0. Schmidt, "Multiple emitter location and signal parameter estimation," in Proc. RADC Spectral Est. Workshop, Oct. 1979, pp. 243-258.

[7] S. U. Pillai, "Array Signal Processing", Springer-Verlag, New York, 1989

[8] T. Shan, M. Wax, and T. Kailath, "On Spatial Smoothing for Direction-of-Arival Estimation of Coherent Signals", IEEE Transactions on Acoustics, Speech and Signal Processing. Vol. 33, No. 4, pp. 80611, Aug. 1985

[9] A. K. Jain, "Fundamentals of Digital Image Processing", Prentice Hall, Englewood Cliffs, NJ, 1989. 